\title{
The Wet Mode Analysis of Rudder System
}

\author{
M.J. Li \\ Institute of Launch Dynamics, \\ Nanjing University of Science and Technology, \\ Nanjing, China \\ X.T. Rui \\ Institute of Launch Dynamics, \\ Nanjing University of Science and Technology, \\ Nanjing, China
}

\begin{abstract}
To calculate a rudder system's vibration characteristic, MSC Nastran was carried out to simulate an underwater model's wet modes based on finite element method (FEM) and virtual mass method. The model was simplified from the rudder system using a torsion spring instead of the transmission parts. The frequencies and mode shapes of the system's first 4 order wet modes were obtained and showed differences in comparison with the dry modes. It could be concluded that the water's effect plays a significant role on rudder system's structural dynamics which should not be neglected in its normal modes analysis.
\end{abstract}

Keywords-rudder; wet mode; MSC Nastran, FEM, virtual mass method.

\section{INTRODUCTION}

For a rudder system, flutter will be induced by incorrect design as the wings of aircraft [1]. Since the strong flutter which leads to violent vibration and structure damage only happens when the speed is higher than 100 knots which is out of marine's speed limit, the flutter problem of the rudder system is often neglected in the early research. But the weak flutter may exist in low speed and it will produce noise in the water which goes against marine's stealth. Thus, it is necessary to study on rudder system's flutter problem. Prior to the flutter analysis, an accurate structural dynamics model should be built [2]. Finite element method (FEM) is widely used because of its ability to obtain high fidelity and reliable solutions for complex geometries with various boundary conditions [3]. Thus, the structural dynamics modelling of the rudder system are performed by the FEM. It should be noticed that the rudder is under the water, therefore, the wet mode analysis of the structure considering fluid's effect is different with the dry mode. The vibrating structure in the fluid is subjected to the effect of fluid's resistance, and a part of the fluid vibrates in coupling with the structure which presents as an added mass in system's mass matrix. Consequently, it is necessary to compute the virtual fluid mass matrix for the underwater system. The method is available in MSC Nastran. Through the normal modes analysis on the finite element model, rudder system's wet modes can be obtained using MSC Nastran.

\section{IITHEORY}

The finite element equation of underwater structure's free vibration is [4]

\author{
L.K. Abbas \\ Institute of Launch Dynamics, \\ Nanjing University of Science and Technology, \\ Nanjing, China \\ H.L. Yu \\ Institute of Launch Dynamics, \\ Nanjing University of Science and Technology, \\ Nanjing, China
}

$$
\left[\boldsymbol{M}+\boldsymbol{M}_{\boldsymbol{A}}\right] \cdot[\ddot{\boldsymbol{u}}]+\left[\boldsymbol{K}+\boldsymbol{K}_{A}\right] \cdot[\boldsymbol{u}]=\{\boldsymbol{0}\}
$$

Where, $\boldsymbol{M}$ and $\boldsymbol{K}$ are the mass and stiffness matrices of the structure respectively. $\boldsymbol{M}_{\boldsymbol{A}}$ and $\boldsymbol{K}_{\boldsymbol{A}}$ are the added mass and stiffness matrices due to water's effect on the structure, respectively. $\boldsymbol{K}_{\boldsymbol{A}}$ could be ignored since it is much lower than $\boldsymbol{K}$, while $\boldsymbol{M}_{\boldsymbol{A}}$ plays an important role which should be mainly considered in the underwater structure's vibration analysis.

The Helmholtz method used by MSC Nastran solves Laplace's Equation by distributing a set of sources over the outer boundary. Each produces a simple solution to the differential equation. By matching the assumed known boundary motions to the effective motion caused by the sources, it may solve a linear matrix equation for the magnitude of the sources. The values of the sources determine the effective pressures and, thereby, the forces on the grid points. Combining all of these steps into a matrix equation results in a virtual mass matrix as derived below $[4,5]$.

If $\sigma_{j}$ is the value of a point source of fluid (units are volume flow rate per area) located at location $r_{j}$, and is assumed acting over an area $A_{j}$, the vector velocity $\dot{u}_{i}$ at any other point $r_{i}$ is

$$
\dot{u}_{i}=\sum_{j} \int_{A_{j}} \frac{\sigma_{j} e_{i j}}{\left|r_{i}-r_{j}\right|^{2}} d A_{j}
$$

Where $e_{i j}$ is the unit vector in the direction from point $j$ to point ${ }^{i}$. Note that the gradient of the vector $\dot{u}_{i}$ is the potential function which satisfies Laplace's Equation on a term by term basis. The other set of necessary equations are the pressures $p_{i}$, 
at any point ${ }^{i}$, in terms of the density $\rho$, sources and geometry, namely

$$
p_{i}=\sum_{j} \int_{A_{j}} \frac{\rho \dot{\sigma}_{j} e_{i j}}{\left|r_{i}-r_{j}\right|} d A_{j}
$$

The results of integrating eqns. (2) and (3) over the finite element surfaces are collected respectively in two matrices, $[\chi]_{\text {and }}[\Lambda]$ where

$$
\{\dot{\boldsymbol{u}}\}=[\chi] \cdot[\sigma]
$$

and

$$
\{\boldsymbol{F}\}=[\Lambda]\{\dot{\boldsymbol{\sigma}}\}
$$

Where $\boldsymbol{F}$ are the forces at the grid points. The matrix $[\Lambda]$ is obtained by integrating eqn. (3). Additional area integration is necessary to convert the pressures to forces. A mass matrix may now be defined using eqns. (4) and (5) as

$$
\{\boldsymbol{F}\}=\left[\boldsymbol{M}_{\boldsymbol{A}}\right] \cdot\{\ddot{\boldsymbol{u}}\}
$$

Where the virtual fluid mass matrix $\left[\boldsymbol{M}_{\boldsymbol{A}}\right]$, is

$$
\left[\boldsymbol{M}_{A}\right]=[\Lambda] \cdot[\chi]^{-1}
$$

\section{MODEL}

Since the entire transmission parts of the rudder system is to provide a torque to the rudder, making the rudder can swing up and down, the whole system (as shown in fig. 1) was simplified into to a torsion spring except the rudder blade [6], the shaft inside the rudder blade was modeled as a beam. The simplified model is shown in fig. 2. In this way, it is more convenient to analyze the rudder system's vibration characteristic and flutter conditions by change the system's parameters.

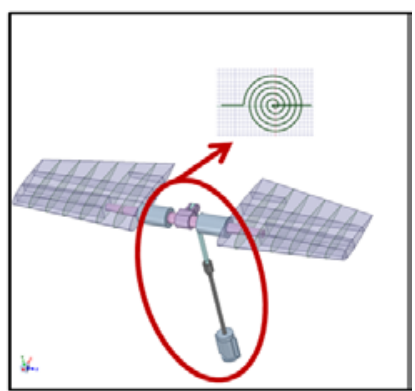

FIGURE I. RUDDER SYSTEM

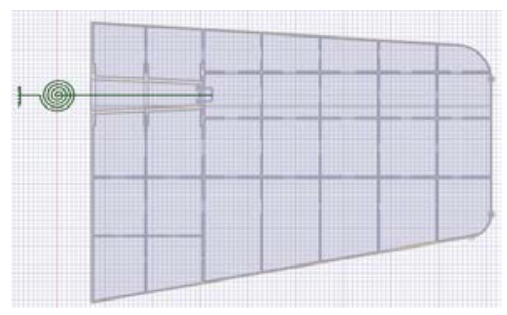

FIGURE II. SIMPLIFIED MODEL

The material of the rudder is steel and the constitutive properties are $E I=2 \times 10^{11} \mathrm{~Pa}, \nu=0.3$ and $\rho=7850 \mathrm{~kg} / \mathrm{m}^{3}$. The computational structural dynamics (CSD) model is constructed in a multi-stage process:

1. Cover: The cover consists of Quad4 (shell element) elements as shown in fig. 3

2. Keel: The keel consists of Quad4 (shell element) elements and Tria3 (shell element) elements as shown in fig. 4, the holes in the keel are not overlooked to make the model as the real rudder.

3. Shaft and the cover of the shaft: As shown in fig. 5, the shaft inside the rudder blade is modeled as a beam consisting of Bar2 (beam element) elements, the shaft's cover inside the rudder consists of Quad4 (shell element) elements. The shaft and the shaft's cover are attached using MPC (RBE2).

4. Transmission part: The transmission part is simplified into a rotational spring which is a Bar (spring element) element, and the spring is attached to the beam using MPC (Rigid) as shown in fig. 5.

5. Boundary Condition: The end of the spring bonded with the beam is fixed by all five degrees of freedom except the rotational one in y direction (along shaft axis' direction). The six degrees of freedom of torsional spring's other end are all fixed. The boundary constraints are shown in fig. 6 .

6. Water inside the rudder blade: Since the rudder is full of water when it is diving underwater, the water will make the rudder heavier and affect the structural dynamics. The Point elements (shown in fig. 7) are built on the nodes of rudder's inside surface that contacts with the inside water, and the inside water's mass is evenly distributed on these point elements.

7. Free surface of the water : The set of input data as follows is used to do the underwater mode analysis [5]

$$
\begin{array}{ll}
\text { Case control: } & \text { MFLUID=1 } \\
\text { Bulk Data: } & \text { PARAM,VMOPT,1 } \\
& \text { MFLUID,1,,300.0,1000.0,11,,N,N } \\
& \text { ELIST,11,10000,THRU,14115 }
\end{array}
$$

Where, $300.0 \mathrm{~m}$ is the depth of the rudder under the water, $1000.0 \mathrm{~kg} / \mathrm{m}^{3}$ is water's density, ELIST set 11 defines the positive faces of elements in the range 10000 through 14115 (the nodes of the rudder that contacts with the outside water) as the structural/fluid boundary. 
8. Parameters: The modal is built according to the real rudder, the thickness of the shell elements are following the real size which are different in many places.

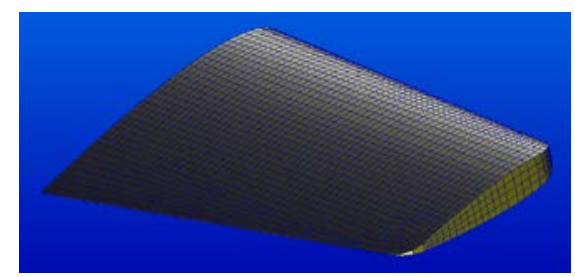

FIGURE III.

HE COVER OF THE RUDDER

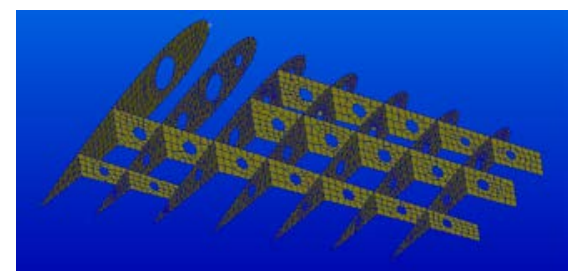

FIGURE IV.

HE KEEL OF THE RUDDER

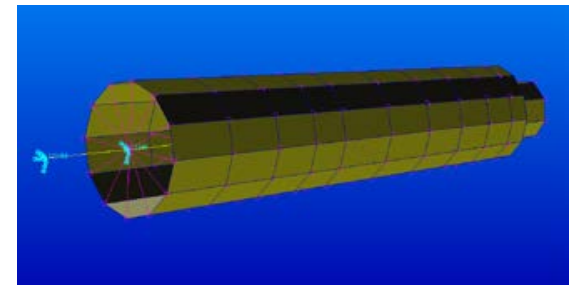

FIGURE V. THE SPRING, SHAFT AND SHAFT'S COVER

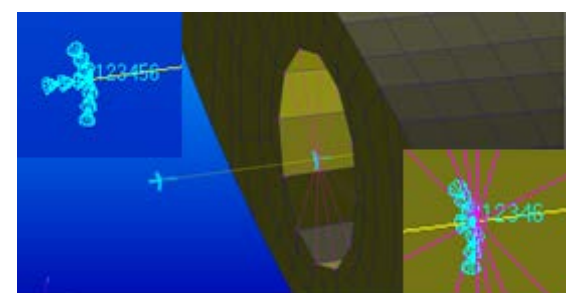

FIGURE VI.

THE BOUNDARY CONDITION

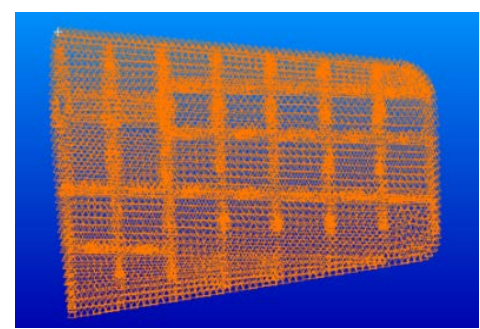

FIGURE VII.

THE POINT ELEMENTS OF WATER'S MASS INSIDE THE RUDDER

\section{RESULT}

The wet modes could be obtained from MSC Nastran. The code of bulk data and case control which defines the fluid/structure interface and the fluid's character and boundaries should be deleted to get the results of dry modes (only considering the mass of the water inside the rudder). The results of the rudder normal modes analysis are given in table 1 and figs. 8 and 9. It showed that the frequencies of the wet modes are lower than the dry modes, the deformations of the dry modes are larger than the wet modes. However, the second order and the third order exchanged because of the effect of water outside the rudder.

TABLEI. The FREQUENCIES OF THE RUdDER

\begin{tabular}{ccccc}
\hline \hline Mode order & 1 & 2 & 3 & 4 \\
\hline Wet mode(Hz) & 13.7 & 24.8 & 35.9 & 68.5 \\
Dry mode(Hz) & 26.1 & 36.9 & 38.6 & 113.3 \\
\hline
\end{tabular}

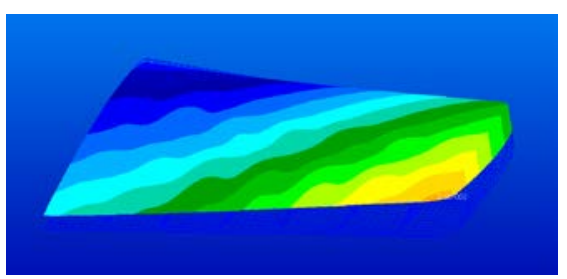

(a) The first order

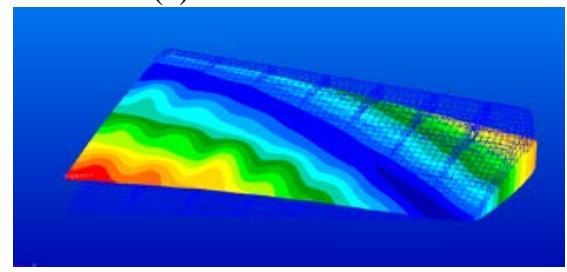

(b) The second order

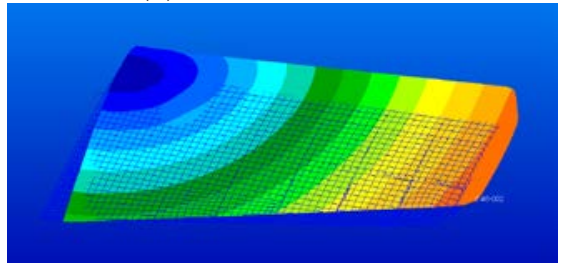

(c) The third order

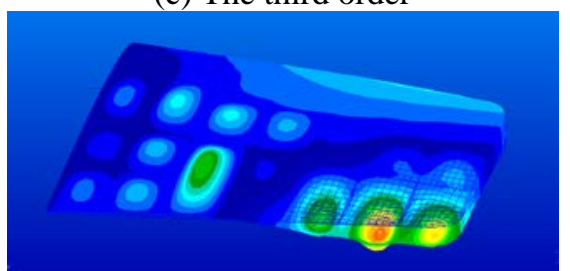

(d) The fourth order

FIGURE VIII. RUDDER'S FIRST FOUR ORDER MODE SHAPES OF THE WET MODES.

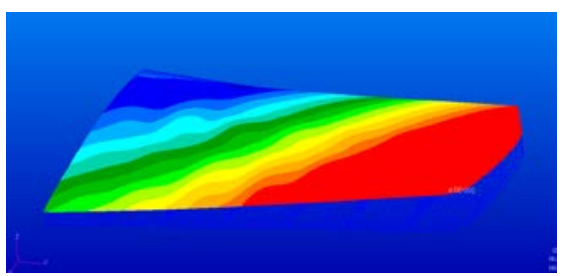

(a) The first order

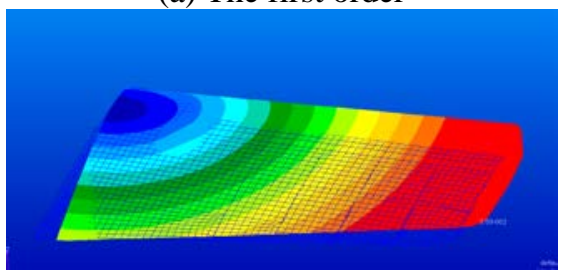


(b) The second order

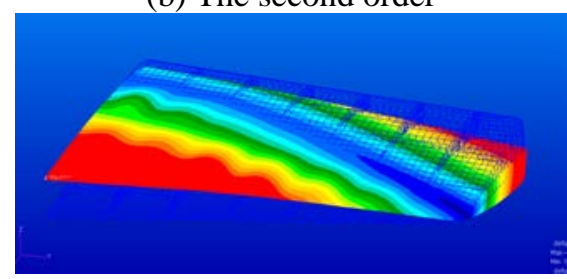

(c) The third order

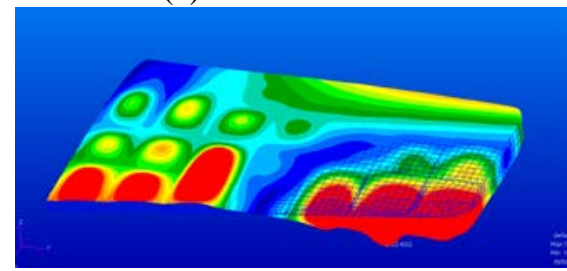

(d) The fourth order

FIGURE IX.

RUDDER'S FIRST FOUR ORDER MODE SHAPES OF THE DRY MODES

\section{VCONCLUSION}

An underwater rudder system's vibration characteristic is calculated. The computational structure dynamic model was built by finite element method of MSC Nastran to simulate the underwater model's wet modes based on virtual mass method. Comparing to the results of the dry modes, the frequencies and deformations of the mode shapes of the wet modes are lower, the second order and the third order exchanged because of the effect of water outside the rudder. From the computational output, it is clear that the water's effect plays a significant role on rudder system's structural dynamics which should be considered in the normal modes analysis prior to the flutter analysis.

\section{ACKNOWLEDGEMENTS}

The research was supported by the Research Fund for the Doctoral Program of Higher Education of China (20113219110025, 20133219110037).

\section{REFERENCE}

[1] Zhang, X. C., Sima, C., \& Wu, Y. S., Low-Speed Flutter Phenomenon of Submarine Rudder and Its Prediction, Journal of Ship Mechanics, 5(1), pp. 70-72, 2001.

[2] Hu, P., Zhao, H. W., Xue, L. P., et al, High-fidelity Modeling and Simulation of Flutter/LCO for All-movable Horizontal Tail with Freeplay, AIAA Atmospheric Flight Mechanics Conference, Portland, 2011.

[3] Nalci, M. O., Aeroservoelastic Modelling of a Missile Control Fin, Master Dissertation, Aeronautical and Aerospace Engineering Department, Middle East Technical University, Ankara, 2013.

[4] Liu, R. F., Huang, R., Zhou, X. R., et al, Contrast Study on Calculation Method for Lower Order Wet Mode of Ship Hull. Ship Engineering, 36(4), pp. 25-28, 2014.

[5] Dynamic Analysis User's Guide, MSC Nastran, 2012.

[6] Lee, I., \& Kimt, S. H., Aeroelastic Analysis of a Flexible Control Surface with Structural Nonlinearity, Journal of Aircraft, 32(4), pp. 868874, 1995. 